\title{
Frequency Dependency of Multi-layer OLED Current Density-voltage Shift and Its Application to Digitally-driven AMOLED
}

\author{
Hyunjong Kim, Suhwan Kim, and Yongtaek Hong* \\ Department of Electrical Engineering and Computer Science, and Inter-university Semiconductor \\ Research Center, Seoul National University, Seoul 151-744, Korea
}

(Received April 13, 2012 : revised May 2, 2012 : accepted May 3, 2012)

\begin{abstract}
We report, for the first time, operation frequency dependence of current density-voltage $\left(\mathrm{J}_{\mathrm{OLED}}{ }^{-}\right.$ $\mathrm{V}_{\mathrm{OLED}}$ ) shift for multi-layer organic light-emitting diodes (OLEDs). When the OLEDs were electrically stressed for 21 hours with $50 \%$ duty voltage pulses at $60,120,240$, and $360 \mathrm{~Hz}$, the $\mathrm{J}_{\mathrm{OLED}}-\mathrm{V}_{\mathrm{OLED}}$ shifts were suppressed by half for $360 \mathrm{~Hz}$ operation compared with $60 \mathrm{~Hz}$ operation, but with little change in emission efficiencies. This frequency dependent $\mathrm{J}_{\mathrm{OLED}}-\mathrm{V}_{\mathrm{OLED}}$ shift is believed to be commonly observed for typical multi-layer OLEDs and can be used to further improve lifetime of digitally-driven active-matrix OLED displays.
\end{abstract}

Keywords: OLED, Frequency, Bias stress, Degradation, Digital driving

OCIS codes : (250.0250) Optoelectronics; (160.4890) Organic materials; (230.3670) Light-emitting diodes

\section{INTRODUCTION}

Recently, digital driving methods have attracted much attention for simplifying pixel structures of active-matrix organic light-emitting diode (OLED) displays (AMOLEDs) [1-4] in comparison with analog counterparts [5-6]. However, in digital driving methods, a driving thin-film transistor (TFT) is generally operated in the linear regime, making pixel brightness degradation more sensitive to a shift in current density-voltage $\left(\mathrm{J}_{\mathrm{OLED}}-\mathrm{V}_{\mathrm{OLED}}\right)$ characteristics of pixel OLEDs. As $\mathrm{J}_{\mathrm{OLED}}-\mathrm{V}_{\mathrm{OLED}}$ characteristics shift with operation time, lifetime of the digitally-driven AMOLED will be further reduced. Therefore, in order to suppress $\mathrm{J}_{\text {OLED }}-\mathrm{V}_{\text {OLED }}$ shifts, pulsed driving or negative biasing methods [7-9] can be additionally combined with digital driving methods because it is well known that $\mathrm{AC}$ driving or negative biasing improves $\mathrm{J}_{\mathrm{OLED}}-\mathrm{V}_{\mathrm{OLED}}$ characteristics [10-12] by affecting redistribution of space charge, movement of ionic impurities, and rotations of permanent dipoles in the typical multilayer OLEDs [13-15]. In addition, for thin film devices where charge trapping and defect creation are dominant degradation mechanisms, improvement of the operation stability has been reported depending on the bias stress fre- quencies although rather low frequencies ranging from 1 to $4 \mathrm{~Hz}$ were used [16].

In this paper, for the first time, we report improvement of operational electrical stability of multi-layer OLEDs depending on operation frequencies. We investigated frequency dependency of shift in current density-voltage ( $\mathrm{JLLED}^{-}$ $\mathrm{V}_{\mathrm{OLED}}$ ) characteristics and proposed that higher frequency operation can improve the lifetime of the digitally-driven AMOLED

\section{FABRICATION AND EXPERIMENTAL WORK}

We used bottom-emission blue fluorescent OLEDs with typical multi-layer structures of indium-tin-oxide (ITO) anode / hole-injection-layer (HIL) / hole-transport-layer (HTL) / doped emissive-layer (EML) / electron-transport-layer (ETL) / lithium-fluoride-aluminum $(\mathrm{LiF} / \mathrm{Al})$ cathode. Although details of the material information cannot be disclosed due to their proprietary properties, thickness and energy band diagram for the fabricated OLED are presented in Fig. 1(a). ITO-patterned glass substrates were treated with ultraviolet

\footnotetext{
*Corresponding author: yongtaek@snu.ac.kr

Color versions of one or more of the figures in this paper are available online.
} 


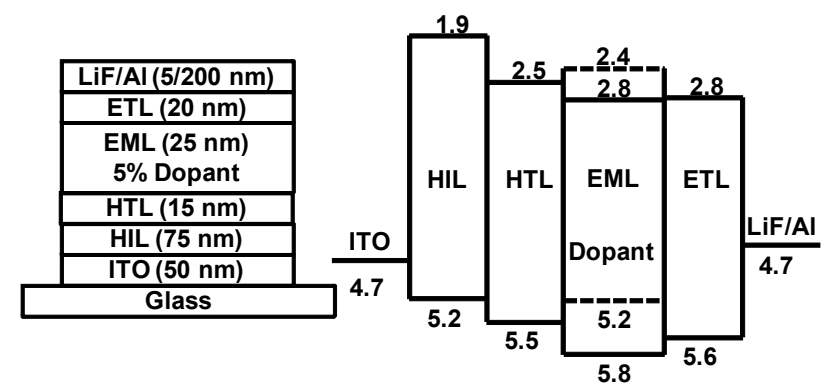

(a)

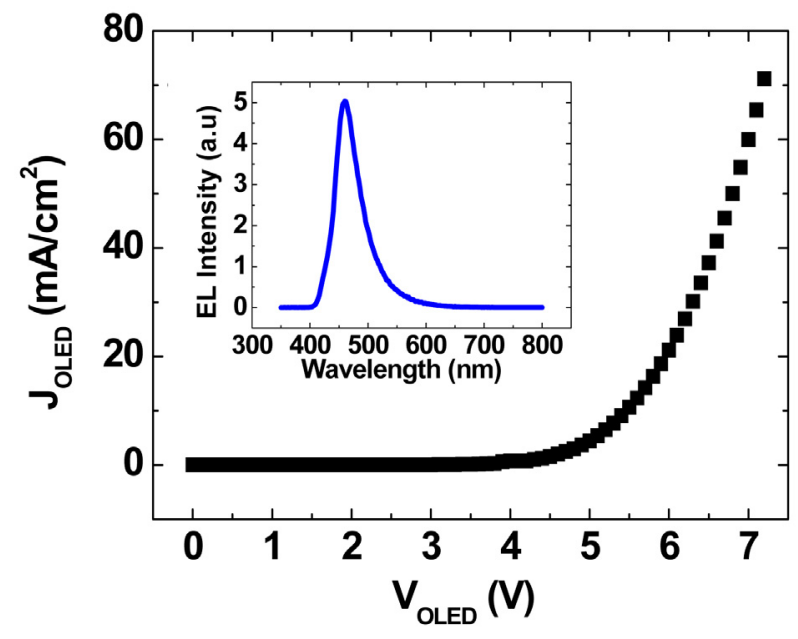

(b)

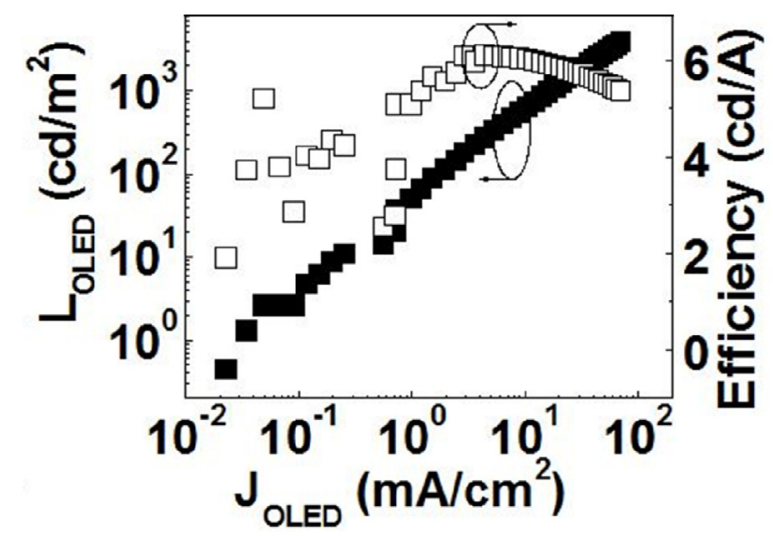

(c)

FIG. 1. (a) Schematic description of structure and energy band diagram, (b) current density-voltage characteristics (electroluminescence spectrum is also included in inset), and (c) efficiency characteristics for the fabricated multi-layer blue OLED.

(UV) ozone for 15 minutes after a conventional cleaning process and then annealed on a hot plate for 10 minutes. After the organic multi-layers and cathode were sequentially deposited without breaking vacuum, the fabricated OLEDs were encapsulated by using glass caps and desiccant. Lightemitting area was $2 \times 2 \mathrm{~mm}^{2}$.

Voltage pulse stress tests were performed by using a semiconductor parameter analyzer, HP $4155 \mathrm{C}$, and a pulse generator unit, HP 41501B. OLED luminance (LoLED) was measured with a luminance meter (Minolta LS-100). We electrically stressed OLEDs by applying voltage pulses with $50 \%$ duty ratio at $60,120,240$, and $360 \mathrm{~Hz}$. Changes in $\mathrm{J}_{\mathrm{OLED}}-\mathrm{V}_{\mathrm{OLED}}$ characteristics and LOLED were monitored every three hours. Since a forming process is typically observed for multi-layer OLEDs at an early stage of the aging process [11], we performed a pre-aging process to make sure that OLEDs show similar initial $\mathrm{J}_{\mathrm{OLED}^{-}} \mathrm{V}_{\mathrm{OLED}^{-}}$ LOLED characteristics and typical aging behavior without any forming processes during the stress test. To further investigate the degradation mechanism, we also applied several negative biases during the voltage pulse stress test. All measurements were performed at room temperature in air.

\section{RESULTS}

Figure 1(b) and 1(c) show $\mathrm{J}_{\mathrm{OLED}}-\mathrm{V}_{\mathrm{OLED}}$ and the electroluminescence spectrum, and $\mathrm{LOLED}^{-} \mathrm{J}_{\mathrm{OLED}}$ and emission efficiency characteristics of the fabricated OLEDs, respectively. We obtained maximum emission efficiency of about $6 \mathrm{~cd} / \mathrm{A}$ and maximum power efficiency of about $4 \mathrm{~lm} / \mathrm{W}$. To accelerate the degradation process of the OLEDs, we selected $7 \mathrm{~V}$ as the high voltage of the voltage stress pulses. Estimated average luminance under this driving condition is about $3000 \mathrm{~cd} / \mathrm{m}^{2}$, which is higher by four times than maximum luminance $\left(700-800 \mathrm{~cd} / \mathrm{m}^{2}\right)$ of blue OLEDs required for commercial mobile AMOLED with full-white brightness of $150 \mathrm{~cd} / \mathrm{m}^{2}$.

Figure 2(a) shows $\mathrm{J}_{\text {OLED }}-\mathrm{V}_{\text {OLED }}$ shifts with stress time $(0$ to 18 hours) for $360 \mathrm{~Hz}$ stress test (solid symbols). Similar $\mathrm{J}_{\mathrm{OLED}}-\mathrm{V}_{\mathrm{OLED}}$ shifts were observed for 60,120 , and $240 \mathrm{~Hz}$ stress tests, and we included $\mathrm{J}_{\mathrm{OLED}}-\mathrm{V}_{\mathrm{OLED}}$ characteristics after 21 hour stress test at each frequency (open symbols) for comparison. In this experiment, higher $V_{\text {peak }}$ is supplied than that for conventional constant voltage driving method by considering current density to compensate the zero luminance in low voltage level of digital driving. As shown in Fig. 2(b), since $50 \%$ duty cycle has been used for digital driving, $2 \times \mathrm{I}_{\text {peak }}\left(\alpha \times \mathrm{V}_{\text {peak }}\right.$ depending on $\mathrm{I}-\mathrm{V}$ characteristics of OLED) must be applied in order to have average luminance that is equal to the luminance for the OLED driven at constant $I_{\text {peak. }}$ Therefore, in typical digital driving, peak voltage or current must be carefully chosen by considering required average luminance and duty cycle. It is also noted that if OLED is driven at its maximum efficiency although peak current is doubled, the high peak current effect on lifetime reduction is relatively small.

As stress time increases, $\mathrm{J}_{\mathrm{OLED}}-\mathrm{V}_{\mathrm{OLED}}$ characteristics shift to the right for all frequencies but in smaller amount for higher frequencies. $\mathrm{J}_{\mathrm{OLED}}-\mathrm{V}_{\mathrm{OLED}}$ shifts are about 0.4 and $0.2 \mathrm{~V}$ (for $50 \mathrm{~mA} / \mathrm{cm}^{2}$ ) after 21 hours stress test at 60 and $360 \mathrm{~Hz}$, respectively. This corresponds to about 50\% suppression in the $\mathrm{J}_{\mathrm{OLED}}-\mathrm{V}_{\mathrm{OLED}}$ shift and about $8 \%$ suppression in 


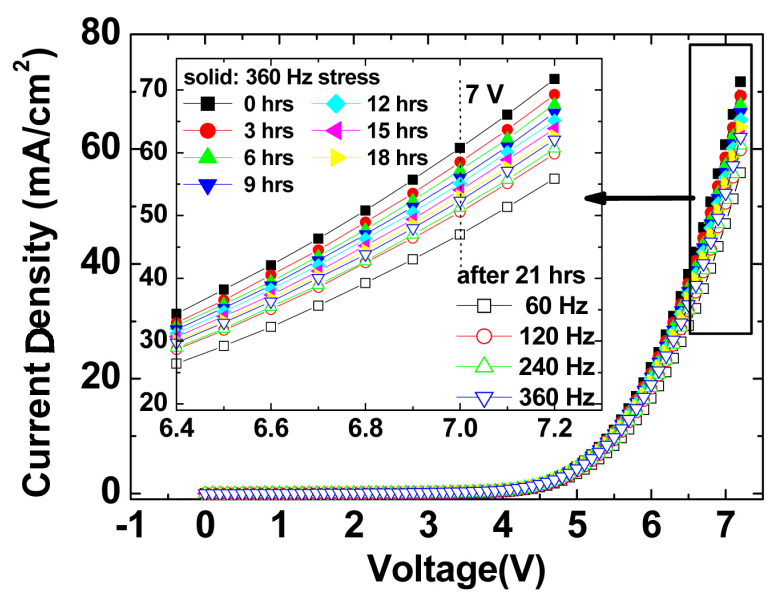

(a)

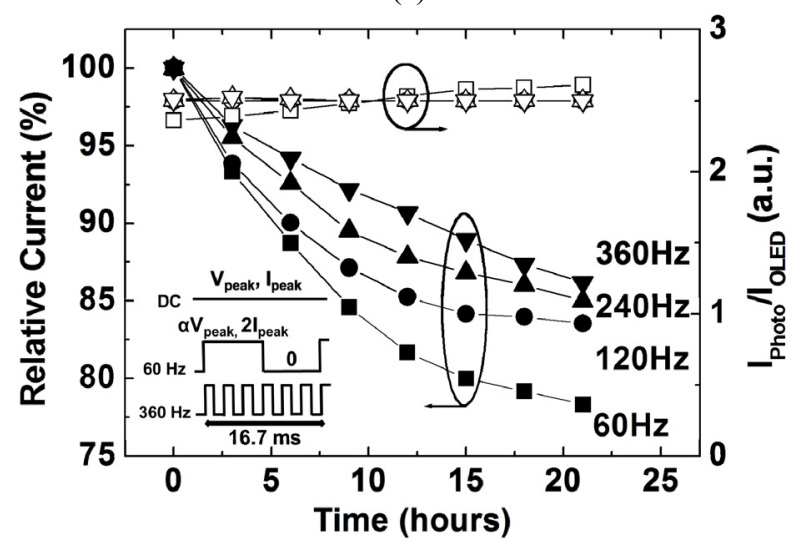

(b)

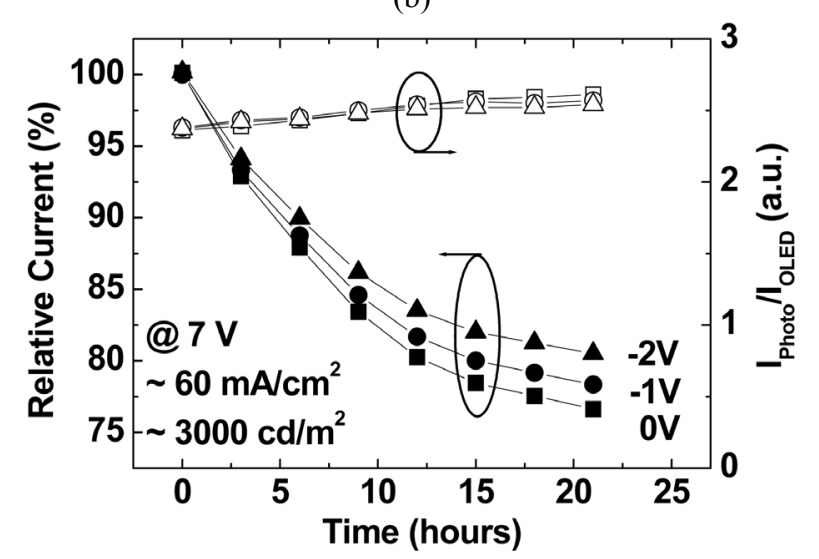

(c)

FIG. 2. (a) Shift in current density-voltage and luminancevoltage characteristics for $360 \mathrm{~Hz}$ stress test (0 to 18 hours) and for $60,120,240$, and $360 \mathrm{~Hz}$ stress test after 21 hours, (b) extracted current and emission efficiency changes at $7 \mathrm{~V}$ for 60, 120, 240, and $360 \mathrm{~Hz}$ stress tests, and (c) extracted current and emission efficiency changes at $7 \mathrm{~V}$ for $60 \mathrm{~Hz}$ stress tests with $0,-1$, and $-2 \mathrm{~V}$ bias conditions

OLED current change at constant voltage of $7 \mathrm{~V}$ as indicated in Fig. 2(a) (dashed line). If we plot changes in current and emission efficiency for the constant voltage with stress time, frequency dependency of electrical degrada- tion can be clearly seen as shown in Fig. 2(b). Since OLED brightness change was monitored with a photodiode during the stress tests, it was recorded as photocurrent in ampere units. Therefore, the emission efficiency is represented as the ratio of the measured photocurrent to the electrical OLED current in Fig. 2(b). Relative current reduction from the initial current level is larger for lower frequency operations. The relative current reached about $86 \%$ and $78 \%$ after 21 hours stress test at 360 and $60 \mathrm{~Hz}$, respectively, while the emission efficiency changes are not much different for all frequencies.

It is speculated that $\mathrm{J}_{\mathrm{OLED}}-\mathrm{V}_{\mathrm{OLED}}$ shift and corresponding relative current reduction were suppressed because operation frequency can affect space charge distribution and movement in our OLEDs, leading to changes in charge or trap accumulation, which is one of the major degradation mechanisms for multi-layer OLEDs [17-18]. To further investigate this effect, we applied negative biases ( -1 and $-2 \mathrm{~V})$ instead of zero voltage in the voltage pulse signals. Figure 2(c) shows changes in relative current and emission efficiency with stress time for $60 \mathrm{~Hz}$ stress test. The relative current was less reduced for driving conditions with negative biases in comparison with the condition with zero bias while the emission efficiency was not much changed during the stress test. These negative bias effects for $\mathrm{J}_{\mathrm{OLED}}-\mathrm{V}_{\text {OLED }}$ characteristics are consistent with the previously reported results and are considered to be an indication of charge redistribution and accumulation in multi-layer OLEDs [14].

Although other degradation mechanisms can play roles during the lifetime test, charge redistribution and accumulation phenomenon can be considered to commonly occur in the course of the degradation process for typical multilayer OLEDs, sometimes depending on stages during the whole lifetime span [19]. In fact, similar frequency dependent $\mathrm{J}_{\mathrm{OLED}}-\mathrm{V}_{\mathrm{OLED}}$ shifts were also observed for other multi-layer red and green OLEDs in our previous study [20]. Therefore, we believe that high frequency operation would give a benefit to lifetime improvement in AMOLED with a conventional digital driving scheme, where one frame time is divided into several sub-frames according to the number of bits representing gray scales [2]. A sub-frame representing the most significant bit (MSB) can be considered to approximately occupy half of one frame time. Since constant voltage is applied during each sub-frame, large degradation and thus, large $\mathrm{J}_{\mathrm{OLED}}-\mathrm{V}_{\mathrm{OLED}}$ shift, is expected during the MSB sub-frame. Therefore, we can divide the MSB subframe into two or three and effectively operate pixel OLEDs at higher frequencies for the MSB signals. Assuming 60 (or 120) Hz display operation, the MSB signal is effectively applied to a pixel OLED at 60 (or 120) Hz. If the MSB sub-frame is divided into two or three and then distributed in one frame time, the MSB signal is considered to be effectively applied to the pixel OLED at 120 (or 240) or 180 (or 360 ) Hz, respectively. Therefore, via sub-division of the MSB signals and possible inclusion of negative biasing schemes, we can suppress pixel OLED J JLED $-V_{\text {OLED }}$ shift 
with operation time, extending lifetime of the digitally-driven AMOLED.

\section{CONCLUSION}

We report the operation frequency dependent $\mathrm{J}_{\mathrm{OLED}}-\mathrm{V}_{\mathrm{OLED}}$ shift in multi-layer OLEDs. When OLEDs were stressed at $360 \mathrm{~Hz}, \mathrm{~J}_{\mathrm{OLED}}-\mathrm{V}_{\mathrm{OLED}}$ shift was suppressed by $50 \%$ and corresponding suppression in current reduction at constant voltage was $8 \%$, but with little change in emission efficiencies, compared with $60 \mathrm{~Hz}$ stress test. Since $\mathrm{J}_{\text {OLED }}-\mathrm{V}_{\text {OLED }}$ shift is directly related to brightness reduction of digitallydriven AMOLED pixel, extended display lifetime is expected when effectively high frequency operation is incorporated into the conventional digital driving scheme.

\section{ACKNOWLEDGMENT}

Authors would like to thank Samsung SDI for OLED devices. This work was supported by Basic Science Research Program through the National Research Foundation of Korea(NRF) funded by the Ministry of Education, Science and Technology(MEST) (Grant No. KRF-2008-331-D00216). Authors would like to thank Mr. Seungjun Chung for valuable discussion and for paper preparation.

\section{REFERENCES}

1. M. Kimura, I. Yudasaka, S. Kanbe, H. Kobayashi, H. Kiguchi, S. Seki, S. Miyashita, T. Shimoda, T. Ozawa, K. Kitawada, T. Nakazawa, W. Miyazawa, and H. Ohshima, "Low-temperature polysilicon thin-film transistor driving with integrated driver for high-resolution light emitting polymer display," IEEE Trans. Elec. Dev. 46, 2282-2288 (1999).

2. M. Mizukami, K. Inukai, H. Yamagata, T. Konuma, T. Nishi, J. Koyama, S. Yamazaki, and T. Tsutsui, "6-bit digital VGA OLED,” Tech. Dig. SID 31, 912-915 (2000).

3. Y. Tanada, M. Osame, R. Fukumoto, K. Saito, J. Sakata, S. Yamazaki, S. Murakami, S. Inose, and N. Miyoshi, "A 4.3-in. VGA (188 ppi) AMOLED display with a new driving method," Tech. Dig. SID 35, 1398-1401 (2004).

4. S. Ono, K. Miwa, and T. Tsujimura, "Active-matrix OLED displays by equally-separated-subframe driving method," Tech. Dig. SID 37, 325-328 (2006).

5. A. Nathan, G. R. Chaji, and S. J. Ashtiani, "Driving schemes for a-Si and LTPS AMOLED displays," J. Display Technology
1, 267-277 (2005).

6. J. Kim, Y. Hong, and J. Kanicki, "Amorphous silicon TFTbased active-matrix organic polymer LEDs," IEEE Elec. Dev. Lett. 24, 451-453 (2003).

7. S. Yujuan, Z. Yi, C. Xinfa, and L. Shiyong, "A simple and effective AC pixel driving circuit for active matrix OLED," IEEE Trans. Elec. Dev. 50, 1137-1140 (2003).

8. Y.-C. Lin and H.-P. D. Shieh, "Improvement of brightness uniformity by AC driving scheme for AMOLED display," IEEE Elec. Dev. Lett. 25, 728-730 (2004).

9. Y. Si, L. Lang, Y. Zhao, X. Chen, and S. Liu, "Improvement of pixel electrode circuit for active-matrix OLED by application of reverse-biased voltage," IEEE Trans. Cir. Sys. 52, 856-859 (2005).

10. D. Zou, M. Yahiro, and T. Tsutsui, "Improvement of currentvoltage characteristics in organic light emitting diodes by application of reversed-bias voltage," Jpn. J. Appl. Phys. 37, L1406-L1408 (1998).

11. X. Liu, W. Li, J. Yu, J. Peng, Y. Zhao, G. Sun, X. Zhao, $\mathrm{Y}$. $\mathrm{Yu}$, and $\mathrm{G}$. Zhong, "Effect of duty ratio of driving voltage on the forming process in aging of organic electroluminescent device,” Jpn. J. Appl. Phys. 37, 6633-6635 (1998).

12. T. Tsujioka, H. Fujii, Y. Hamada, and H. Takahashi, "Driving duty ratio dependence of lifetime of tris (8-hydroxy-quinolinate) aluminum-based organic light-emitting diodes," Jpn. J. Appl. Phys. 40, 2523-2526 (2001).

13. J. Shen, D. Wang, E. Langlois, W. A. Barrow, P. J. Green, C. W. Tang, and J. Shi, "Degradation mechanisms in organic light emitting diodes," Synth. Met. 111-112, 233-236 (2000).

14. D. Zou and T. Tsutsui, "Voltage shift phenomena introduced by reverse-bias application in multilayer organic light emitting diodes," J. Appl. Phys. 87, 1951-1956 (2000).

15. M. Nakai, H. Fujii, T. Tsujioka, Y. Hamada, and H. Takahashi, "Degradation of organic layers of organic light emitting devices by continuous operation," Jpn. J. Appl. Phys. 41, 881-884 (2002).

16. N. Mohan, K. S. Karim, S. Prakash, and A. Nathan, "Stability issues in digital circuits in amorphous silicon technology," Proc. Canadian Conference on Electrical and Computer Engineering 1, 13-16 (2001).

17. S. J. Martin, G. L. B. Verschoor, M. A. Webster, and A. B. Walker, "The internal electric field distribution in bilayer organic light emitting diodes," Org. Electron. 3, 129-141 (2002).

18. D. Y. Kondakov, "Direct observation of deep electron traps in aged organic light emitting diodes," J. Appl. Phys. 97, 024503-1 024503-5 (2005).

19. Z. D. Popovic and H. Aziz, "Reliability and degradation of small molecule-based organic light-emitting devices (OLEDs)," IEEE J. Select. Topics Quantum Electron. 8, 362-371 (2002).

20. H. Kim, S. Kim, Y. Hong, S. W. Chang, D. Lee, D. S. Jeong, and H. K. Chung, "Frequency dependence of OLED voltage shift degradation," Proc. IMID 7, 1108-1111 (2007). 